10 Montani D, Bergot E, Günther S, et al. Pulmonary arterial hypertension in patients treated by dasatinib. Circulation 2012; 125: 2128-2137.

11 Savale L, Chaumais MC, Cottin V, et al. Pulmonary hypertension associated with benfluorex exposure. Eur Respir J 2012; 40: 1164-1172.

12 Souza R, Humbert M, Sztrymf B, et al. Pulmonary arterial hypertension associated with fenfluramine exposure: report of 109 cases. Eur Respir J 2008; 31: 343-348.

\title{
The thoracic cage becomes flattened in the progression of pleuroparenchymal fibroelastosis
}

\author{
To the Editor:
}

Pleuroparenchymal fibroelastosis (PPFE) was first reported by FRANKEL et al. [1]. PPFE can occur without any aetiology or underlying diseases (idiopathic PPFE), or with underlying diseases or conditions. Idiopathic PPFE has been listed as one of the rare idiopathic interstitial pneumonias (IIPs) in the revised international multidisciplinary consensus classification of IIPs [2]. The natural history of PPFE is variable, some are slowly progressive and others sometimes show rapid deterioration resulting in poor prognosis, like idiopathic pulmonary fibrosis (IPF). Idiopathic pulmonary upper lobe fibrosis (PULF), first proposed by AMITANi et al. [3], is currently considered to be almost identical to idiopathic PPFE [1, 4, 5], which is now globally accepted as a representative nomenclature for this disorder. Therefore, we use the term PPFE to describe the same disease as PULF.

AMITANi et al. [3] recognised a characteristic constitution in patients with PPFE: they are slender and their thoracic cage is flattened, i.e. the ratio of the anteroposterior diameter of the thoracic cage (APDT) to the transverse diameter of the thoracic cage (TDT) is abnormally lower than in normal populations. Herein, we have provisionally named this deformity of the thoracic cage as "flat chest". Other investigators have also noticed this deformity in idiopathic PPFE [6-8]. Flat chest may result from a congenital disposition or may be an acquired deformity of the thoracic cage associated with fibrosing upper lung lobes.

We assessed the APDT/TDT ratio in patients with PPFE. Subsequently, we examined whether flat chest became more pronounced during the progression of the disease. Based on clinical files from our hospitals, eight patients with PPFE in whom the APDT/TDT ratio had been measured twice with an interval of $\geqslant 1.87$ years using chest computed tomography (CT) were retrospectively enrolled. TDT was determined as the longest transverse diameter of the thoracic cage measured parallel to a line (line \#) along the rearmost points of the 6th thoracic vertebra in the horizontal section of the chest CT (fig. 1a). APDT was determined as the longest distance of the anteroposterior dimension of the thoracic cage measured perpendicular to the line along the rearmost points of the 6th thoracic vertebra (line \#). In addition, TDT and APDT were also defined as the distances from the inside of a rib to the inside of the opposite rib. If APDT in one hemithorax was different to the APDT in the other hemithorax, a mean value was adopted. We also compared the change in forced vital capacity (FVC) during the interval between the two measurements of APDT/TDT. The first FVC was defined as the FVC measured around the first measurement of APDT/TDT, with a time lag $<4 \%$ of the interval between the two measurements of APDT/TDT. The second FVC was defined as the FVC measured around the second APDT/TDT, with intervals of 1.42-4.67 years between the two measurements of FVC.

In case 2, flat chest became more pronounced after an interval of 5.27 years: APDT/TDT decreased from 0.592 to 0.509 and FVC decreased from 3540 to $1120 \mathrm{~mL}$ (fig. 1). In eight patients, the APDT/TDT became significantly lower after intervals of 1.87 to 5.27 years (paired t-test, $p=0.003$ ), during which FVC was remarkably decreased in six patients (table 1 ).

Barrel chest is a well-known acquired deformity of the thoracic cage in patients with advanced pulmonary emphysema. It is possible that a long-standing increase in intrathoracic pressure, caused by overinflated lungs associated with obstructive ventilatory impairment, leads to an expansion of the thoracic cage into 

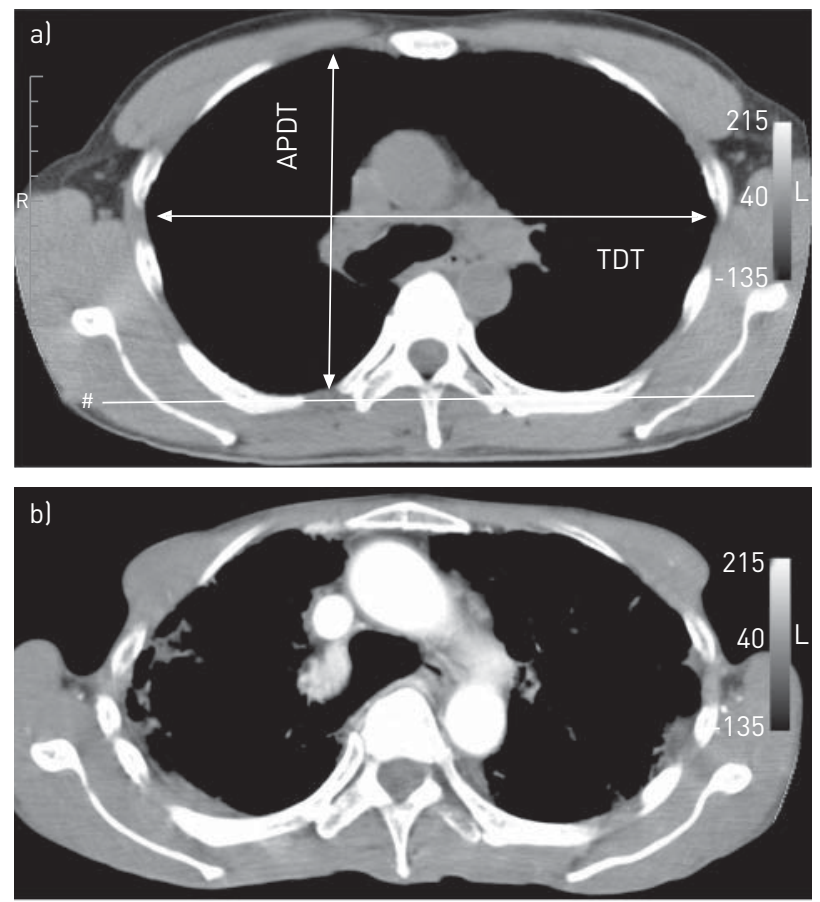

FIGURE 1 Chest computed tomography scan from a 55-year-old male with pleuroparenchymal fibroelastosis (case 2) at the level of the 6th thoracic vertebra a) on admission and b) 5.27 years later. The anteroposterior diameter of the thoracic cage (APDT)/transverse diameter of the thoracic cage (TDT) ratio decreased after 5.27 years. Note the space present between the anterior edge of the ascending aorta and the anterior chest wall in (a). The anterior edge of the ascending aorta was attached to the anterior chest wall after 5.27 years. TDT was determined as the longest transverse diameter of the thoracic cage measured parallel to a line (\#) that runs along the rearmost points of the bilateral 6th ribs in the horizontal section of the CT.

barrel chest. In contrast, we have demonstrated that a long-standing fibrosing process in the bilateral upper lobes led to the contraction of the thoracic cage to flat chest in patients with PPFE. However, the wide variety of the observation periods, due to the retrospective design, is a limitation of this study.

To compare the severity of flat chest in the same condition, the APDT/TDT was measured at the level of the 6th thoracic vertebra, which includes the fibrosing bilateral upper lobes. In case 1, however, the APDT/TDT did not decrease after an interval of 4.39 years. Because the patient's flat chest was already very advanced at the time of the first measurement, there seemed to be no further apparent progression of the deformity thereafter.

AMITANi et al. [3] speculated that the deformity of the thoracic cage in idiopathic PPFE results from a congenital disposition. However, we found that the thoracic cage became flattened in the deteriorated course of PPFE, although the possibility that flat chest was already present before the onset of PPFE cannot be excluded.

NAKASONE et al. [9] reported two PPFE patients who had undergone allogenic stem cell transplantation; the thoracic cage was flattened in one of the patients. We have shown that three of the eight patients with flat chest had various underlying diseases, such as ulcerative colitis, post-irradiation therapy and lung transplantation. Such findings may support the hypothesis that flat chest is not caused by a hereditary disposition; rather, it seems to be an acquired deformity that is secondary to the progressively decreasing volume of fibrosing upper lobes. However, it is also possible that flattened chest caused by fibrosis results in immobilisation of the upper lungs which further advances fibrosis.

In PPFE, both FVC and total lung capacity are decreased, whereas the ratio of forced expiratory volume in $1 \mathrm{~s} / \mathrm{FVC}$ is increased, which is similar to IPF. However, fibrotic collapse of the upper lobes leads to the compensatory over inflation of the lower lobes. This results in an increase in the ratio of residual volume/ total lung capacity [10], which is a specific functional impairment not usually seen in IPF. Reciprocal mechanical vectors produced by shrinkage of upper lobes and over inflation of lower lobes might be reflected in the specific physical finding of flat chest. 


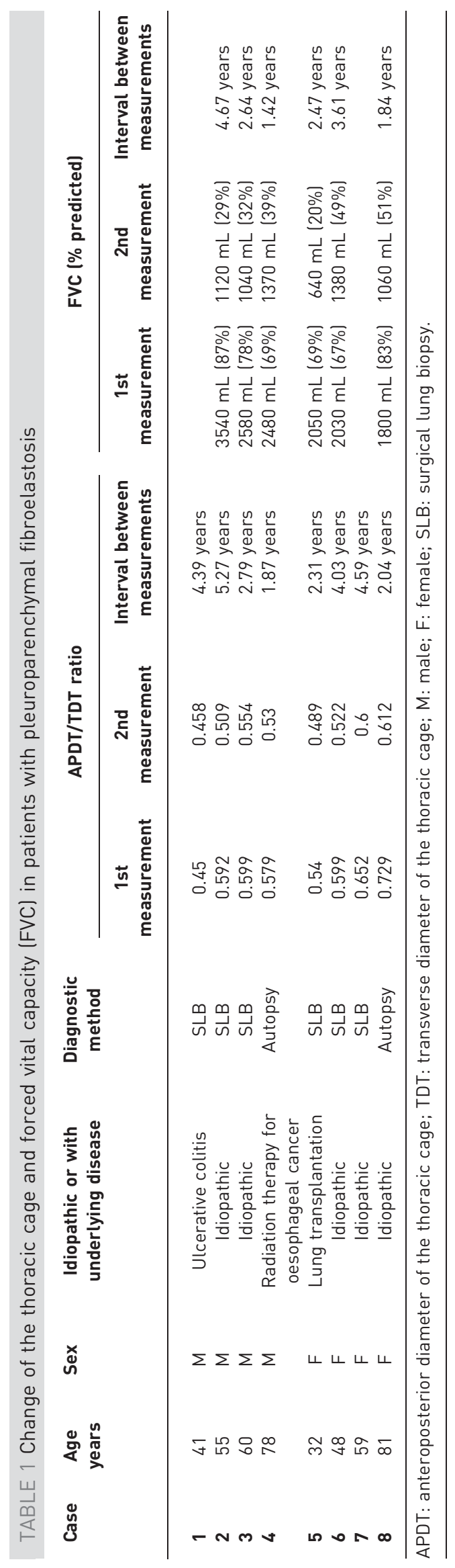


Taishi Harada ${ }^{1}$, Yuji Yoshida ${ }^{1}$, Yasuhiko Kitasato ${ }^{2}$, Nobuko Tsuruta ${ }^{3}$, Kentaro Wakamatsu ${ }^{4}$, Takako Hirota ${ }^{1}$, Makoto Tanaka ${ }^{1}$, Naoki Tashiro ${ }^{1}$, Hiroshi Ishii ${ }^{1}$, Motokimi Shiraishi ${ }^{1}$, Masaki Fujita ${ }^{1}$, Nobuhiko Nagata ${ }^{5}$ and Kentaro Watanabe ${ }^{1}$

${ }^{1}$ Dept of Respiratory Medicine, Fukuoka University School of Medicine, Fukuoka, Japan. ${ }^{2}$ Dept of Respiratory Medicine, Kurume Dai-ichi Hospital, Kurume, Japan. ${ }^{3}$ Dept of Respiratory Medicine, Hamanomachi Hospital, Fukuoka, Japan. ${ }^{4}$ Dept of Respiratory Medicine, National Hospital Organization, Omuta National Hospital, Omuta, Japan. ${ }^{5}$ Dept of Respiratory Medicine, Fukuoka University Chikushi Hospital, Fukuoka, Japan.

Correspondence: K. Watanabe, Dept of Respiratory Medicine, Fukuoka University School of Medicine, Fukuoka 8100180, Japan. E-mail: watanabe@fukuoka-u.ac.jp

Received: Sept 162013 | Accepted after revision: Oct 142013

Support statement: This work was partly supported by a grant to the Diffuse Lung Diseases Research Group (Dept of Respiratory Medicine, Jichi Medical University, Tochigi, Japan) from the Ministry of Health, Labour and Welfare (Tokyo, Japan).

Conflict of interest: None declared.

Provenance: Submitted article, peer reviewed.

\title{
References
}

Frankel SK, Cool CD, Lynch DA, et al. Idiopathic pleuroparenchymal fibroelastosis: description of a novel clinicopathologic entity. Chest 2004; 126: 2007-2013.

2 Travis WD, Costabel U, Hansell DM, et al. An Official American Thoracic Society/European Respiratory Society statement: update of the international multidisciplinary consensus classification of the idiopathic interstitial pneumonias. Am J Respir Crit Care Med 2013; 188: 733-748.

Amitani R, Niimi A, Kuze F. [Idiopathic pulmonary upper lobe fibrosis]. Kokyu 1992; 11: 693-699.

Reddy TL, Tominaga M, Hansell DM, et al. Pleuroparenchymal fibroelastosis; a spectrum of histopathological and imaging phenotypes. Eur Respir J 2012; 40: 377-385.

5 Watanabe K, Nagata N, Kitasato Y, et al. Rapid decrease in forced vital capacity in patients with idiopathic pulmonary upper lobe fibrosis. Respir Investig 2012; 50: 88-97.

6 Kobayashi Y, Sakurai M, Kushiya M, et al. [Idiopathic pulmonary fibrosis of the upper lobe: a case report]. Nihon Kokyuki Gakkai Zasshi 1999; 37: 812-816.

7 Iesato $\mathrm{K}$ Ogasawara $\mathrm{T}$, Masuda $\mathrm{A}$, et al. [Idiopathic pulmonary upper lobe fibrosis: clinical and pathological features]. Rinsho Houshasen 2005; 50: 13-25.

8 Morimoto A, Mochizuki Y, Nakahara Y, et al. [Case of idiopathic pulmonary upper lobe fibrosis]. Nihon Kokyuki Gakkai Zasshi 2010; 48: 944-949.

9 Nakasone E, Bando M, Nakao T, et al. [Pleuroparenchymal fibroelastosis in patients with pulmonary disease after bone marrow transplantation]. Nihon Kokyuki Gakkai Zasshi 2012; 1: 562-566.

10 Kusagaya $\mathrm{H}$, Nakamura $\mathrm{Y}$, Kono $\mathrm{M}$, et al. Idiopathic pleuroparenchymal fibroelastosis: consideration of a clinicopathological entity in a series of Japanese patients. BMC Pulm Med 2012; 12: 72.

\section{Specific inhalation challenge tests for occupational asthma in Europe: a survey}

\author{
To the Editor:
}

Asthma is described as "occupational" if it has been induced by an agent encountered in the workplace. The avoidance of further exposure to the causative agent often results in resolution of the asthma, especially if it can be achieved soon after the onset of symptoms [1]. Consequently, occupational asthma is one of the very few types of asthma that are potentially curable.

The implications of this include the importance of early recognition of occupational asthma and of accurate identification of the causative agent. A variety of methods are available to make a diagnosis [2], among which specific inhalation challenge (SIC) testing is generally considered to be the reference standard [3-5]. In this context, SIC testing is the controlled exposure of a patient, under laboratory conditions, to an agent 